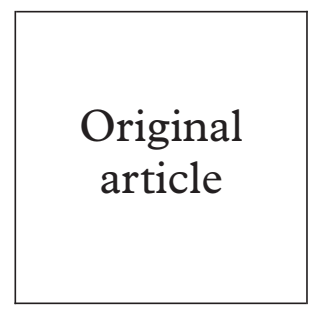

\section{Predictors of syphilis seroreactivity and prevalence of HIV among street recruited injection drug users in Los Angeles County, 1994-6}

\author{
Javier López-Zetina, Wesley Ford, Mark Weber, Stefi Barna, Theresa Woerhle, \\ Peter Kerndt, Edgar Monterroso
}

Objectives: To describe HIV prevalence and the association between syphilis incidence and sexual and drug injection risk behaviours in a cohort of street recruited injecting drug users (IDUs) in Los Angeles County, between 1994 and 1996.

Methods: During the study period, 513 street recruited African-American and Latino IDUs were screened for syphilis and antibodies to HIV. Subjects were administered a risk behaviour survey at baseline and followed up at 6 month intervals for 18 months with repeated interviews and serological screening. Rate ratios were used to examine associations between syphilis incidence and demographic characteristics and risk behaviours. A proportional hazard model was used to identify predictors of syphilis incidence independent of demographic characteristics.

Results: $74 \%$ of the sample were male, $70 \%$ African-American, $30 \%$ Latino; and the median age was 43 years. Overall baseline serological prevalence of HIV was $2.5 \%$ and of syphilis $5.7 \%$. None of the participants were co-infected for HIV and syphilis at baseline or at any of the 6 month follow ups. Among 390 eligible IDUs retained for analysis of incidence data, the overall syphilis incidence was 26.0 per 1000 person years. Higher syphilis incidence was found for women compared with men $(\mathrm{RR}=2.70 ; 95 \% \mathrm{CI} 1.60,4.55)$, and for those 44 years of age or younger compared with those 45 years of age and older ( $R R=2.26 ; 95 \%$ CI 1.25, 4.08). African-Americans were more likely to be syphilis incident cases when compared with Latinos, although the difference did not reach statistical significance ( $R R=1.27 ; 95 \%$ CI $0.72,2.23)$. In bivariate analysis, risk behaviours significantly associated with higher syphilis incidence included injection of cocaine, "speedball" and heroin, "crack" smoking, recency of first injection event, backloading of syringes, injecting with others, exchanging drugs or money for sex, multiple sex partners, and non-heterosexual sexual preference. Variables that significantly predicted syphilis infection at follow up in the multivariate analysis included multiple sex partners (RR=7.8; 95\% CI 2.4, 25.0), exchanging money for sex $(\mathrm{RR}=3.0 ; 95 \% \mathrm{CI} 0.9,9.6)$, and recent initiation to injection drug use ( $\mathrm{RR}=4.6 ; 95 \%$ CI 1.1, 18.8).

Conclusion: Syphilis transmission among IDUs in Los Angeles County remains a serious public health concern, particularly among IDUs who engage in trading of sex for money or drugs. Although low, the prevalence of HIV observed in this study constitutes a serious concern because of the potential for expanded HIV transmission in this susceptible population of IDUs with high syphilis incidence. Enhanced case finding screening efforts and prevention of transmission of sexually transmitted infections should specifically target hard to reach IDUs and their sexual partners.

(Sex Transm Inf 2000;76:462-469)

Keywords: syphilis incidence; injection drug users; HIV prevalence

W Ford

$M$ Weber

T Woerhle

P Kerndt

Los Angeles County Department of Health Services, Sexually Transmitted Disease Program, Los Angeles, California, USA

S Barna

Centers for Disease Control and

Prevention, Atlanta,

Georgia, USA

E Monterroso

Correspondence to: Dr Javier López-Zetina, Department of Health

Science, California State University, Long Beach,

1250 Bellflower Blvd, Long

Beach, CA 90840, USA

jlopezze@csulb.edu

Accepted for publication

12 September 2000

\section{Introduction}

Multiple studies have reported the concurrent association between injection drug use, HIV infection, and other sexually transmitted diseases. ${ }^{1-4}$ Syphilis, in particular, is increasingly seen as a marker for high sexual risk behaviour among injection drug users (IDUs). ${ }^{5}$ Previous studies of syphilis among IDUs have suggested that while high prevalence of syphilis and low HIV prevalence are often found in clinical settings, in drug treatment centres the reverse pattern of moderately high prevalence of HIV and low prevalence of syphilis may be more common. ${ }^{6}$ In addition to these differences in patterns of co-morbidity among IDUs depending on recruitment settings, pronounced regional variation in the prevalence of these infections has been observed in this rela- tively comparable high risk group..$^{7-9}$ Despite these differences and frequent atypical serological presentation, syphilis serology screening among IDUs provides a valuable opportunity to further our understanding on the epidemiological patterns of indirect transmission of HIV and other STDs from the injecting to the noninjecting population. While sustained spread of syphilis has not been reported in the United States in recent years, periodic outbreaks of the infection as well as localised high prevalence foci remain an important public health problem. ${ }^{10}$ Further, the epidemiological and clinical problems posed by its association with HIV have important implications for the prevention and clinical management of the coinfection. ${ }^{11}{ }^{12}$ The role of parenteral transmission of HIV in the spread of the epidemic among IDUs has been examined extensively, 
yet little is known about other bloodborne and sexually transmitted infection interactions in low HIV prevalent areas. ${ }^{13}$ Prevalence estimates of HIV infection among IDUs in Los Angeles County are considerably lower than in the east coast. ${ }^{1415}$ In areas of low HIV prevalence, the presence of other markers of parenteral and sexual HIV risk transmission provides important means to understand the spread of HIV and to establish prevention measures. ${ }^{16}{ }^{17}$

A steady overall decline in nationwide rates of primary and secondary syphilis has been reported during the 1990 s, yet, many metropolitan urban areas continue to experience increasing number of syphilis cases and dramatic disparities still exist among some populations. ${ }^{12}$ For example, the US syphilis rate for African-Americans was almost 60 times greater than for non-Hispanic white people during this time period. ${ }^{18}$ In Los Angeles County, the 3 year average syphilis incidence from 1992 to 1994 was $6.0 / 10,^{5}$ and the county ranked fourth in the average crude case rate of syphilis incidence reported statewide between 1992 and $1994 .{ }^{19}$ Moreover, primary syphilis among African-Americans in Los Angeles County is 20 times more prevalent than among white people, and three times more prevalent among Latinos than white people. ${ }^{20}$ With the growing contribution of IDU to the spread of HIV/ AIDS, drug users, particularly IDUs, are thus an important target for preventing HIV and syphilis transmission because these infections share similar risk factors, and because IDUs are a recognised behavioural population linking high risk individuals to the non-injecting population.

This paper reports on factors associated with syphilis incidence in a cohort of IDUs, followed over an 18 month period in south central Los Angeles, and discusses the implications of these findings for HIV and STD prevention and control.

\section{Methods}

SAMPLE

Participants were recruited as part of a longitudinal study with a primary focus on HIV and STDs risk behaviours. Recruitment took place at Los Angeles County street locations where IDUs are known to congregate. The study was part of a larger, national, multisite study of risk behaviours and risk of HIV seroconversion among IDUs. ${ }^{9}$ Dynamic recruitment by word of mouth and street outreach, and active follow up of participants began in August 1994 and continued until August 1996. Open enrolment of eligible participants continued throughout the first 12 months of follow up. Subjects in the selected neighbourhoods were invited into the study using a protocol based on a semistructured screening interview to determine eligibility. Only individuals who had injected drugs within the past 12 months and who were not enrolled in a drug treatment programme at the time of the initial interview were eligible to participate. The three recruitment venues were located in south central Los Angeles areas where the pre- dominant racial/ethnic composition is AfricanAmerican and Latino. Peer and/or indigenous outreach workers trained in survey administration and certified in HIV counselling and phlebotomy screened, enrolled, and interviewed participants in a especially equipped mobile testing unit. After the baseline interview, participants were actively followed at 6 month intervals with repeated risk behaviour interviews and serological screening.

Follow up of participants was based on a protocol to ensure ongoing contact with participants. Features of this protocol included periodic updates at 3 month intervals of participants' baseline locator information (current address, telephone number, contact person, etc). Updates were obtained by mail, in person, or by having participants call a toll-free phone number. Attempts to locate subjects who failed to return for scheduled follow up appointments included ongoing searches at social services agencies, county jails, and state prisons, as well as searches of the Los Angeles County's death certificate databases. Fifty four of the 886 follow up interviews were conducted at county and state detention facilities. By one evaluation measure of this tracking protocol (number of participants who returned at least once after baseline ascertainment into the study), follow up was moderately successful with a retention rate of $85 \%$. However, only $37 \%$ of the participants were able to complete all three scheduled follow up interviews.

After written informed consent was obtained, the survey questionnaire was administered and serum specimens were obtained by venepuncture or fingerstick. To minimise socially desirable responses to risk behaviour questions, participants were provided with pretest counselling following survey administration. The standardised survey questionnaire consisted of detailed questions on drug use and sexual behaviour practices during the previous 6 months. As part of the HIV pretest counselling, participants were offered condoms and bleach kits free of charge. They also were reimbursed with a small stipend of $\$ 20$ to offset travel expenses. Study subjects were also encouraged to return to the interview site the week following their interview to receive their serological test results and additional risk reduction counselling, and were given the option of calling the toll-free telephone number to set up an appointment and receive their test results. In order to defray any other transportation expenses incurred while receiving their results, participants were offered an additional $\$ 5$ stipend. Participants were informed that although HIV test results were to remain confidential, state law required that a positive syphilis test would be reported to the Los Angeles County Department of Health Services. Upon completion of the intake interview and phlebotomy, subjects were given appointment cards to update their locator information at 3 month intervals and to return for their follow up visit. 
SEROLOGY

Syphilis status was determined by serological evidence. Specimens reactive to both nontreponemal test (non-treponemal Rapid Plasma Reagin-Venereal Disease Research Laboratory, RPR-VDRL (Hardy Laboratory) and treponemal test (microhaemagglutination-T pallidum, MHA-TP (Miles Laboratory)), were considered syphilis cases. By this criterion, subjects were determined as incident cases if serological evidence indicated any reactive RPR-VDRL test with a titre of 1:1 or higher at any follow up visit, confirmed with a reactive MHA-TP tests, and non-reactive RPR-VDRL and MHA-TP tests, and no history of syphilis reported at baseline. The syphilis screening research protocol at the Los Angeles County Public Health Laboratory consisted of a simplified algorithm whereby specimens reactive to RPR-VDRL tests were then confirmed by the MHA-TP test; and no MHA-TP testing was performed on sera from participants who tested non-reactive on the RPR-VDRL test. Ascertainment of syphilis was carried out exclusively by serology screening, and no clinical information was collected in this study. However, study subjects were asked whether they have previously been diagnosed with syphilis and whether or not treatment was received. Given the absence of clinical information, incident syphilis cases could not be staged accurately, although based on the serological data, date of reactive tests, and surveillance definitions cases may be considered early latent cases according to self reported history of syphilis and their midpoint of seroconversion (median 8.6 months; range 5.1-18.2 months). ${ }^{21}$ In addition to the assessment of syphilis seroreactivity, serum specimens were tested for the presence of antibodies to HIV-1 by enzyme linked immunosorbent assay (ELISA), and confirmatory western blot (OTC Laboratories).

\section{ANALYSIS METHODS}

The incidence of syphilis infection was estimated as incidence density-that is, the number of new infections divided by total person years of follow up. The dynamic cohort study design resulted in variable lengths of follow up; therefore, incidence was calculated as the number of new syphilis infections divided by 1000 person years of observation. The seroconversion date was estimated as the midpoint between the baseline negative result and the first positive result. Subjects who failed to return for any given scheduled follow up appointments contributed person year data up to their most current follow up return visit (if any), and the corresponding 6 month interval risk behaviour data were used in conjunction with the most recent test result. Participants with only baseline data were excluded from incidence tabulation but the data analysis methodology utilised all available time data by including in the analysis all participants with at least two data points - that is, baseline and any follow up visit. In addition, five participants with serological evidence of treated syphilis at baseline were also retained in the incidence cohort but their serological profile remained unchanged at follow up.
Demographic variables and risk behaviour variables were collapsed to permit a meaningful analysis of the data. With a median of 43 years, participants in this study formed an older cohort of IDUs, and baseline age was classified in two age groups ( $\leqslant 44 v \geqslant 45$ years). Other demographic variables were categorised as follows: marital status (married $v$ not married), educational status (high school graduate or beyond $v$ less than high school), homelessness, and sources of most income in the past 6 months (illicit income-that is, theft, drug sales, sex trade $v$ legal full or part time job or welfare assistance). Similarly, risk and sexual behaviours ascertained at follow up were stratified according to these categories: injection of any drug in the past 6 month (yes $v$ no), "speedball "and heroin injection frequency (never $v$ once daily $v$ more than once daily), cocaine injection frequency (once daily or less $v$ never), "crack" smoking, backloading of syringes (sometimes $v$ never), recency of first injection ( $\leqslant 5 v>5$ years), age at first injection ( $\leqslant 25 v \geqslant 26$ years), sites of "shooting up" (transient which includes abandoned building, shooting galleries, car, dealers' apartment $v$ non-transient which includes own or spouse's apartment or friend's/relatives's place), and injecting with other people (always $v$ sometimes $v$ never).

Self reported sexual risk behaviours at follow up were categorised as follows: multiple sex partners ( $\geqslant$ two partners $v \leqslant$ one partner, range of first category two to 210 , mean 11.8 ), and exchanging drug, or money for sex (yes $v$ no). Self reported sexual orientationascertained at baseline-was dichotomised in two groups (behavioural sexual group A) composed of heterosexual IDUs, and (behavioural sexual group B) comprising men who have sex with men, bisexual men, and lesbian IDUs. Risk behaviours significantly associated in the bivariate analysis were further examined in a Cox proportional hazards model to identify factors independently associated with syphilis incidence. Interval censoring of the data was utilised to account for participants lost to follow up (that is, study subjects who never returned after their baseline ascertainment into the study), participants with missing baseline serological data, and baseline prevalence cases. Statistical analyses were performed with SAS for Windows 6.10 .

\section{Results}

Five hundred and fifty nine IDUs were recruited from the street venues in Los Angeles County from August 1994 to October 1996. Owing to the extremely small sample size of participants who were white $(3.8 \%)$ or who reported "other" race/ethnic category (3.3\%), the analyses described here were restricted to African-Americans and Latinos (513/559). Eighty five per cent of baseline eligible study subjects (436/513) completed at least one follow up visit, $65 \%$ completed at least two visits, and $37 \%$ completed three follow up visits. Participants lost to follow up did not differ significantly by race, age, marital, or educational status although they were more 
likely to be male and homeless. Seventy four per cent of the sample were male, the median age was 43 years (range 18-70 years), 70\% identified themselves as African-American, and $36 \%$ as homeless. Approximately three in five $(63 \%)$ reported regular part time or full time employment as their main source of income during the preceding 6 months while the rest engaged in illicit activities to obtain money (that is, theft, drug sales, sex trade, etc). At baseline, $60 \%$ of participants reported an educational attainment of high school or beyond. Most participants (99\%) reported heroin as the injection drug of choice, followed by speedball $(63 \%)$ and cocaine $(34 \%)$, and $74 \%$ reported smoking crack in the past 6 months. More than half of the participants were polydrug users, with $52 \%$ reporting use of at least two injection drugs and smokable crack.

Table 1 Serological prevalence of syphilis and HIV at baseline among street recruited injection drug users in Los Angeles County, 1994-6

\begin{tabular}{|c|c|c|c|c|}
\hline \multirow[b]{2}{*}{ Demographic characteristics } & \multicolumn{2}{|l|}{ Syphilis } & \multicolumn{2}{|l|}{$H I V$} \\
\hline & $\begin{array}{l}\text { Cases/total } \\
\text { sample }\end{array}$ & $\%$ Positive & $\begin{array}{l}\text { Cases/total } \\
\text { sample }\end{array}$ & $\%$ Positive \\
\hline Overall prevalence & $28 / 487$ & 5.7 & $13 / 513$ & 2.5 \\
\hline \multicolumn{5}{|l|}{ Sex: } \\
\hline Female & $12 / 128$ & $9.3 \ddagger$ & $4 / 137$ & 2.9 \\
\hline Male & $16 / 359$ & 4.4 & $9 / 376$ & 2.3 \\
\hline \multicolumn{5}{|l|}{ Age (years): } \\
\hline$\leqslant 44$ & $23 / 294$ & $7.8 \ddagger$ & $9 / 308$ & 2.9 \\
\hline$\geqslant 45$ & $5 / 193$ & 2.5 & $4 / 205$ & 1.9 \\
\hline \multicolumn{5}{|l|}{ Race: } \\
\hline African-American & $22 / 336$ & 6.5 & $12 / 360$ & 3.3 \\
\hline Latino & $6 / 151$ & 3.9 & $1 / 153$ & 0.6 \\
\hline \multicolumn{5}{|l|}{ Educational status: } \\
\hline$<$ High school & $15 / 198$ & 7.5 & $4 / 207$ & 1.9 \\
\hline$\geqslant$ High school & $13 / 289$ & 4.4 & $9 / 306$ & 2.9 \\
\hline \multicolumn{5}{|l|}{ Marital status: } \\
\hline Married & $7 / 94$ & 7.4 & $1 / 101$ & 0.9 \\
\hline Not married & $21 / 393$ & 5.3 & $12 / 412$ & 2.9 \\
\hline \multicolumn{5}{|l|}{ Homeless: $\dagger$} \\
\hline Yes & $12 / 180$ & 6.6 & $5 / 184$ & 2.7 \\
\hline No & $16 / 303$ & 5.2 & $8 / 325$ & 2.4 \\
\hline \multicolumn{5}{|l|}{ Source of most income: ${ }^{\star}+$} \\
\hline Illicit sources & $15 / 185$ & 8.1 & $5 / 192$ & 2.6 \\
\hline Regular employment & $13 / 301$ & 4.3 & $8 / 320$ & 2.5 \\
\hline
\end{tabular}

* See text for definition.

$\dagger$ Total varies because of missing data.

$\neq \mathrm{p}<0.05$.

Table 2 Syphilis incidence among injection drug users in Los Angeles County, 1994-6

\begin{tabular}{|c|c|c|c|c|c|}
\hline Demographic characteristics & $\begin{array}{l}\text { Cases/total } \\
\text { sample }\end{array}$ & $\begin{array}{l}\text { Incidence } \\
\text { rate/1000 } \\
\text { person years }\end{array}$ & $\begin{array}{l}\text { Rate } \\
\text { ratio }\end{array}$ & $95 \% C I$ & $p$ Value \\
\hline Overall incidence & $12 / 390$ & 26.0 & - & $19.4,32.3$ & \\
\hline \multicolumn{6}{|l|}{ Sex: } \\
\hline Female & $6 / 106$ & 48.1 & 2.7 & $1.60,4.55$ & $<0.01$ \\
\hline Male & $6 / 284$ & 17.8 & & & \\
\hline \multicolumn{6}{|l|}{ Age (years) } \\
\hline$\leqslant 44$ & $9 / 227$ & 34.2 & 2.2 & $1.25,4.08$ & $<0.01$ \\
\hline$\geqslant 45$ & $3 / 163$ & 15.1 & & & \\
\hline \multicolumn{6}{|l|}{ Race: } \\
\hline African-American & $9 / 269$ & 27.8 & 1.2 & $0.72,2.23$ & $>0.05$ \\
\hline Latino & $3 / 121$ & 21.8 & & & \\
\hline \multicolumn{6}{|l|}{ Educational status: } \\
\hline$\geqslant$ High school & $8 / 233$ & 29.5 & & & \\
\hline$<$ High school & $4 / 157$ & 20.8 & 0.7 & $0.40,1.23$ & $>0.05$ \\
\hline \multicolumn{6}{|l|}{ Marital status: } \\
\hline Not married & $10 / 318$ & 26.7 & 1.2 & $0.68,2.08$ & $>0.05$ \\
\hline Married & $2 / 72$ & 22.4 & & & \\
\hline \multicolumn{6}{|l|}{ Homeless: } \\
\hline Yes & $3 / 183$ & 31.6 & 1.2 & $0.75,2.17$ & $>0.05$ \\
\hline No & $9 / 306$ & 24.6 & & & \\
\hline \multicolumn{6}{|l|}{ Source of most income: ${ }^{\star}$} \\
\hline Illicit sources & $8 / 105$ & 67.3 & 5.7 & $3.30,9.85$ & $<0.01$ \\
\hline Regular employment & $4 / 282$ & 11.8 & & & \\
\hline
\end{tabular}

^See text for definition.
BASELINE SEROLOGY

Overall prevalence of syphilis, and HIV at baseline by demographic characteristics is shown in table 1. At baseline, the prevalence of syphilis was two times greater than that of HIV. None of the participants was co-infected with HIV and syphilis. In general, female IDUs exhibited a greater prevalence than males for the two serological outcome measures, with syphilis prevalence among females twice that of males. By race/ethnicity, African-Americans showed a higher prevalence when compared with Latino participants. Among white IDUs the prevalence of syphilis was $11 \%(2 / 18)$ but no incidence was observed in this racial/ethnic group. Subsequently, they were excluded from further analysis because of their unstable small sample size. By reported source of income, those who reported illicit sources of income showed a twofold difference in prevalence of syphilis when compared with participants with regular employment. People who considered themselves homeless showed a greater prevalence of syphilis and HIV, although the differences were less pronounced.

SYPHILIS INCIDENCE

Syphilis incidence by selected demographic characteristics is shown in table 2 . Three hundred and ninety participants were retained in the analysis of syphilis incidence after exclusion of participants lost to follow up (77 out of 513 cases with only baseline data), subjects with incomplete syphilis serological data (20 out of 436 cases with no serum specimen for syphilis testing), and syphilis prevalent cases who did not experience a repeated syphilis episode (26 out of 416 cases). The median length of follow up was 8.6 months among syphilis incident cases, and 17.0 months among non-incident cases. The cumulative syphilis incidence during the study period was $3.1 \%$. With 461.1 person years of observation calculated from 390 participants, overall incidence was 26.0 cases per 1000 person years. Women, those under 44 years of age, African-Americans, homeless people, and IDUs who reported illicit sources of income were more likely to be incident cases, although race/ethnicity and homeless status comparisons failed to reach statistical significance.

Among injection risk behaviours, injecting with other people, injecting in transient and/or public places, as well as recent initiation to injection use were significantly associated with a greater risk of becoming infected with syphilis (table 3). Among heroin users, a gradient of greater heroin use and greater risk of becoming infected with syphilis was observed with a rate ratio of 2.9 for those who injected more than once daily, and 1.2 for those who injected once daily or less, compared with those who did not inject heroin during the preceding 6 months. However, most participants were polydrug users and no discernible pattern of risk was observed when any use of speedball, cocaine, and heroin was examined. Independent of drug of choice, injection drug use was significantly associated with syphilis, although the association was only marginally significant $(R R=$ 
1.5 ; 95\% C.I. 0.84, 2.75). Similarly, crack smoking was also associated with greater risk for syphilis infection; however, when crack smoking was analysed independent of injection use, new infections were observed only among those who reported the concurrent use of injection drugs and crack smoking, and no infections were observed among those who reported crack smoking exclusively.

In general, indicators of high risk drug injection behaviours (shooting up in public places, injecting with other people, recent initiation to injection drug use) were associated with increased risk for syphilis, but the only

Table 3 Syphilis incidence among injection drug users by self reported IDU risk behaviours in the past 6 months, Los Angeles County, 1994-6

\begin{tabular}{|c|c|c|c|c|c|}
\hline Injection risk behaviours & $\begin{array}{l}\text { Cases/total } \\
\text { sample }\end{array}$ & $\begin{array}{l}\text { Cases } / 1000 \\
\text { person years }\end{array}$ & $\begin{array}{l}\text { Rate } \\
\text { ratio }\end{array}$ & $95 \% C I$ & $p$ Value \\
\hline Overall incidence & $12 / 390$ & 26.0 & - & $19.4,32.3$ & \\
\hline \multicolumn{6}{|l|}{ Injected any drug: } \\
\hline Yes & $11 / 343$ & 27.3 & 1.5 & $0.84,2.75$ & $>0.05$ \\
\hline No & $1 / 42$ & 17.9 & 1.0 & & \\
\hline \multicolumn{6}{|l|}{ Speedball injection: } \\
\hline More than daily & $1 / 26$ & 35.8 & 1.1 & $0.73,1.92$ & $>0.05$ \\
\hline Daily or less & $2 / 113$ & 15.4 & 0.5 & $0.27,0.93$ & $<0.01$ \\
\hline Never & $9 / 246$ & 30.2 & 1.0 & & \\
\hline \multicolumn{6}{|l|}{ Cocaine injection: } \\
\hline Daily or less & $3 / 52$ & 52.5 & 2.3 & $1.42,3.71$ & $<0.01$ \\
\hline Never & $9 / 331$ & 22.8 & 1.0 & & \\
\hline \multicolumn{6}{|l|}{ Heroin injection: } \\
\hline More than daily & $8 / 187$ & 39.2 & 2.9 & $1.60,5.23$ & $<0.01$ \\
\hline Daily or less & $3 / 143$ & 16.8 & 1.2 & $0.60,2.54$ & $>0.05$ \\
\hline Never & $1 / 56$ & 13.5 & 1.0 & & \\
\hline \multicolumn{6}{|l|}{ Crack smoking: } \\
\hline Sometimes & $9 / 200$ & 39.1 & 3.0 & $1.66,5.51$ & $<0.01$ \\
\hline Never & $3 / 190$ & 12.9 & 1.0 & & \\
\hline \multicolumn{6}{|l|}{ Backloaded syringe:* } \\
\hline Sometimes & $4 / 72$ & 47.2 & 2.1 & $1.33,3.58$ & $<0.01$ \\
\hline Never & $7 / 273$ & 21.6 & & & \\
\hline \multicolumn{6}{|l|}{ Recency of first injection: } \\
\hline 5 years or less & $3 / 22$ & 128.7 & 6.2 & $4.18,9.42$ & $<0.01$ \\
\hline More than 5 years & $9 / 368$ & 20.5 & & & \\
\hline \multicolumn{6}{|l|}{ Sites of shooting up: } \\
\hline Transient & $3 / 81$ & 32.5 & 1.3 & $0.78,2.23$ & $>0.05$ \\
\hline Non-transient & $9 / 308$ & 24.5 & & & \\
\hline \multicolumn{6}{|c|}{ Injected with other people: } \\
\hline Always & $2 / 58$ & 32.4 & 1.8 & $1.03,3.26$ & $<0.05$ \\
\hline Sometimes & $8 / 240$ & 28.2 & 1.6 & $0.88,2.89$ & $>0.05$ \\
\hline Never & $2 / 89$ & 17.6 & & & \\
\hline
\end{tabular}

* Total varies because of missing data.

Table 4 Syphilis incidence among injection drug users by self reported sexual risk behaviour in the past 6 months, Los Angeles County, 1994-6

\begin{tabular}{|c|c|c|c|c|c|}
\hline Sexual risk behaviours & $\begin{array}{l}\text { Cases/total } \\
\text { sample }\end{array}$ & $\begin{array}{l}\text { Cases } / 1000 \\
\text { person years }\end{array}$ & $\begin{array}{l}\text { Rate } \\
\text { ratio }\end{array}$ & $95 \% C I$ & $p$ Value \\
\hline Overall incidence & $12 / 390$ & 26.0 & - & $19.4,32.3$ & \\
\hline \multicolumn{6}{|l|}{ Multiple sex partners: } \\
\hline Yes & $7 / 120$ & 50.2 & 3.2 & $1.89,5.54$ & $<0.001$ \\
\hline No & $5 / 269$ & 15.5 & & & \\
\hline \multicolumn{6}{|c|}{ Exchanged money/drugs for sex: } \\
\hline Yes & $5 / 55$ & 77.7 & 4.4 & $2.76,7.12$ & $<0.001$ \\
\hline No & $7 / 335$ & 17.5 & & & \\
\hline \multicolumn{6}{|l|}{ Sexual orientation: } \\
\hline Group B^ & $4 / 22$ & 187.4 & 10.3 & $7.0,15.2$ & $<0.001$ \\
\hline Group A† & $8 / 367$ & 18.1 & & & \\
\hline
\end{tabular}

*IDU men who have sex with men, and bisexual and lesbian IDUs.

tHeterosexual IDUs.

Table 5 Risk factors for syphilis seroconversion among injection drug users in Los Angeles County, 1994-6

\begin{tabular}{|c|c|c|c|c|c|c|}
\hline \multirow[b]{2}{*}{ Risk factor } & \multicolumn{3}{|c|}{ Unadjusted ratio } & \multicolumn{3}{|c|}{ Adjusted ratio * } \\
\hline & $H R$ & $95 \% C I$ & $p$ Value & $H R$ & $95 \% C I$ & $p$ Value \\
\hline Multiple sex partners & 8.0 & $2.5,25.4$ & $\leqslant 0.01$ & 7.8 & $2.4,25.0$ & $\leqslant 0.01$ \\
\hline Recent initiation to IDU & 6.7 & $1.7,25.4$ & $\leqslant 0.01$ & 4.6 & $1.1,18.8$ & $\leqslant 0.05$ \\
\hline Exchanged money for sex & 3.0 & $0.9,9.4$ & $<0.05$ & 3.0 & $0.9,9.6$ & 0.06 \\
\hline
\end{tabular}

HR = hazard ratio

$95 \% \mathrm{CI}=95 \%$ confidence intervals

*Adjusted for sex, race, and age group. association between syphilis incidence and actual needle sharing was observed among those reporting syringe backloading $(R R=2.18$; $95 \%$ C.I. $1.3,3.5)$, a proxy measure of frequent sharing of drug paraphernalia.

The mean number of sexual partners during the preceding 6 months, reported by syphilis prevalent and incident cases, was 13.7 and 43.0 partners, respectively. In contrast, the mean number among syphilis-free cases after follow up was two partners. Behaviours associated with sex trade (exchanging money or drug for sex) and having multiple sex partners (number of partners $>$ one partner) showed an increased risk for syphilis (table 4). By self reported sexual orientation disclosed at baseline, behavioural sexual group B (homosexual and bisexual men and lesbian women) showed a 10 -fold increased risk for syphilis when compared with the behavioural sexual group B (heterosexual IDUs). Of the four incident cases from behavioural sexual group B, one identified himself as a homosexual male, two as bisexual women, and one as a transsexual biological male. Only one of the incident cases (included in the tabulation of incidence) reported a history of syphilis, and having been treated for it in the past 6 months.

Injection and sexual risk behaviours identified as significant correlates of syphilis incidence in the bivariate analysis were further examined in a multivariate analysis which included time to first infection in the model. A Cox proportional hazards model with interval censoring at the time of the last visit, and baseline demographic characteristics (sex, age, and race), dates of baseline, and last serological screening or first occurrence of a syphilis event, and separate risk behaviour factors was utilised to observe the independent association between risk behaviours and syphilis. Time to first event was defined as the number of months elapsed from a negative baseline screening to first occurrence of a syphilis infection. The strength of the association was determined by hazard ratios and corresponding 95\% confidence intervals. Multiple sex partners $(\mathrm{HR}=7.8 ; 95 \%$ C.I. $2.4,25.0)$, exchanging money for sex $(\mathrm{HR}=3.0 ; 95 \%$ C.I. 0.9, 9.6), and recent initiation to injection drug use (HR $=4.6 ; 95 \%$ C.I. $1.1,18.8)$ appeared as predictors of incident syphilis infection independent of demographic characteristics in the multivariate model (table 5). Predictors in this model were further examined by adding interaction terms to the regression model. Two interaction terms were evaluated separately, one encompassing number of sexual partners and sexual orientation; and a second interaction term that examined number of sexual partners and sex trade. Both models with the interaction terms failed to reach statistical significance ( $p<0.67$ and $p<0.55$, respectively).

\section{Discussion}

The primary finding is that injection drug users recruited through street based outreach in Los Angeles County have a high incidence of syphilis. The incidence of syphilis reported in similar populations elsewhere in the United 
States is markedly lower that the one reported for this IDU cohort. For example, a similar study of IDUs recruited at a methadone maintenance programme in New York found a syphilis incidence of $2.9 / 10^{3}$ person years among IDUs who remained HIV-free during the study period, ${ }^{22}$ in contrast with the Los Angeles incidence of $26.0 / 10^{3}$ person years. The prevalence of early latent syphilis prevalence in the two Los Angeles County health districts where the study was conducted was $97.2 / 10^{5}$ and $59.6 / 10^{5}$ in 1993 , substantially higher prevalence than the county average of $19.3 / 10^{5}$ for the same year. ${ }^{20}$ Although countywide syphilis have been on the decline since 1989, syphilis incidence appears to be increasing in smaller, local communities within certain county areas, a pattern also observed in other large metropolitan areas. ${ }^{23}$ Undiagnosed syphilis infections may be considerable, ${ }^{24}$ particularly in hard to reach, high risk populations like IDUs. Asymptomatic clinical profile of primary syphilis infections, ${ }^{25}$ and lack of ready access to health care may contribute to the rise in syphilis and other bloodborne diseases, especially in areas with inadequate health infrastructure to identify cases and provide treatment.

In the context of the observed twofold increase in syphilis in the general US population from 1985 to $1990,{ }^{26}$ sex differences in syphilis incidence were consistent with other studies that showed a greater likelihood for infection with syphilis among female IDUs, ${ }^{27}$ and more specifically, among female IDUs who engaged in exchanging money and/or drug for sex. ${ }^{28}{ }^{29}$ The results of the multivariate analysis, however, suggest that new syphilis infections were associated with sexual risk factors like trading money for sex, and total number of sex partners, independent of sex. In this respect, it may be argued that the observed greater likelihood of syphilis incidence among women is influenced primarily by IDU mediated risky behaviours, particularly in closeknit networks of injection drug users. ${ }^{30}$ In a population of IDUs with a homogeneous high risk profile, the role of drug mediated increased exposure to HIV and STDs may comprise one of the most important contributing factor to the spread of syphilis and other STDs among women. Significant progress in the prevention and treatment of syphilis has been made over the past decades; however, the number of IDU related and sexually transmitted HIV infections among women continues to rise steadily, and renewed efforts are needed to understand patterns of co-morbidity and transmission of both HIV and syphilis. Our data corroborate the well established finding that sexual contact, particularly that associated with commercial sex, plays a primary part in the spread of syphilis among IDUs, with $T$ pallidum transmission particularly higher among IDUs who smoke crack. ${ }^{31}$ Multiple studies have previously reported the relation between crack smoking and greater risk for sexually transmitted infections, ${ }^{32}$ yet little is known about the specific interaction of crack smoking and injection drug use among polydrug users. However, the inde- pendent contribution of crack use to greater risk for syphilis and HIV among IDUs who engaged in paid sex remains an area for increased efforts to reduce risk. Clearly, prevention efforts must go beyond the provision of sterile injection equipment and include a prevention programme addressing the needs of polydrug users.

Although HIV prevalence in this population of IDUs was low when compared to that reported during the same period in other areas of the United States, particularly the east coast, the high incidence of syphilis suggests that conditions exist for rapid spread of HIV in this group. Prevalence and incidence levels of STDs may be seen as precursors of HIV infection when increased transmission of the HIV virus is experienced in a susceptible population. Neither HIV seroconversion nor HIV and syphilis co-morbidity were observed during the follow up span of the study in this cohort of IDUs. The reported lack of knowledge of syphilis exposure among incident syphilis cases, along with the high number of sexual partners reported, raises serious concerns about the potential for rapid horizontal spread of these infections to non-IDUs. ${ }^{31}$

Several studies, including the present one, have examined the relation between syphilis as a marker for greater risk for HIV seroconversion, ${ }^{33}$ and there is increasing evidence that co-morbidity with syphilis and bloodborne infections is common among IDUs, and IDUs with one of these serological markers infections show a greater likelihood of carrying the other markers. ${ }^{33} 34$ However, the short span of the present study might have resulted in inconclusive data about whether syphilis is an appropriate HIV marker. ${ }^{35} 36$

The apparent paradox of low HIV prevalence, rare HIV seroconversion, and high syphilis incidence among IDUs in Los Angeles County reported in this study may be the results of markedly different IDU milieu and social context where injection drug behaviour takes place when compared to other regions in the United States. This study's cohort of street recruited IDUs was an older group and despite their continued injection drug use and high prevalence of injecting drug behaviours, they may represent a cohort of "survivors" who on account of their low density networks and low geographic mobility have remained unexposed to the HIV virus. While shooting galleries were an ubiquitous features of the IDU milieu in the east coast, IDUs in Los Angeles reported little use of such venues. Only $7 \%$ in our sample reported injecting in shooting galleries in past 6 months. Some studies have suggested that in low HIV prevalence areas exposure to IDUs from high prevalence areas may be better predictor of seroconversion than specific IDU behaviours. $^{37}$ In contrast with the low HIV prevalence among IDUs in the districts where the study was conducted, syphilis appears to have taken a greater foothold in these areas. As mentioned above, the magnitude in the difference in 3 year average syphilis incidence in the specific health districts where the study was 
conducted and the rest of the county was twofold and threefold.

Study limitations should be considered when interpreting our findings. Firstly, our sampling strategy was based on convenience recruitment of study participants at three sites in Los Angeles County; thus, generalising these results to the larger population of IDUs in the area (estimated to fluctuate between 80000 and $200000)^{8}$ is not possible. However, given the well known difficulties of obtaining probability based samples from this population; the ethnographic, targeted recruitment protocol of this study provides data on a hidden population not easily accessible by random sampling mechanisms. Secondly, substantial losses to follow up in this cohort may bias the data and lower our estimates because those participant lost to follow up were more likely to be homeless and male. The present data, however, fill an important gap in our knowledge concerning IDUs, HIV, and STDs, as previous studies have been primarily based on subjects recruited at drug treatment centres, ${ }^{32}{ }^{38}$ public health clinics, ${ }^{35}$ or institutionalised populations. ${ }^{3940}$ Further, as the contribution of IDU to the spread of the HIV/AIDS epidemic continues to grow, understanding the role of indirect exposure to HIV remains a critical factor for the establishment of STDs and HIV prevention measures. Another significant caveat in this study is that syphilis serology alone presents important limitations for the diagnosis of syphilis and remains even more problematic owing to the varied serological response among infected individuals who inject illicit drugs, ${ }^{1141}$ and the limitations of treponemal tests to differentiate between new infections and those previously treated for syphilis. ${ }^{42}$ Owing to the lack of additional clinical information among syphilis cases in our study, it may be argued that IDUs with reactive syphilis serology at follow up might have been the result of a false positive test (as evidenced by the weak titre reactions of less than $1: 4$ in three out of every four incident cases), and positive treponemal tests resulting from previous exposure to and/or treated syphilis.

Despite these limitations, findings from this study support the notion that given the potential increased susceptibility to HIV infection among IDUs with syphilis reported in other studies, ${ }^{12} 29$ reduction of high risk sexual behaviour and of risky injection drug use should be incorporated in innovative STD prevention programmes. Targeted prevention, particularly among IDUs not accessing traditional drug treatment programmes or those lacking access to community STD clinics and facilities, should be an integral component of these efforts. Estimates of unidentified syphilis cases in the United States are high, ${ }^{24}$ and the rise in syphilis observed among residents in inner cities ${ }^{43}$ calls for renewed screening efforts to decrease the spread of STDs and other infectious diseases in disadvantaged communities. Tailored interventions that provide condom and bleach kits to IDUs should become an integral component of preventive efforts aimed at the control of HIV and STDs in the community. Syphilis case finding among IDUs, particularly those not in treatment, could be enhanced by utilising creative community based outreach programmes that provide testing and preventive counselling to this hidden population.

1 Centers for Disease Control. Continuing increase in infectious syphilis, United States. MMWR Morb Mort Wkly Rep 1988;37:35-8.

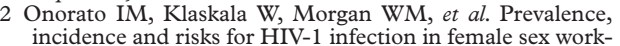
ers in Miami, Florida. $\mathcal{F}$ Acquir Immune Defic Syndr 1995;9: 395-400.

3 Zeldis JB, Jain S, Kuramoto IK, et al. Seroepidemiology of viral infections among intravenous drug users in northern California. West f Med 1992;156:30-5.

4 Levine OS, Vlahov D, Koehler J, et al. Seroepidemiology of hepatitis B virus in a population of injecting drug users. Association with drug injection patterns. Am $\mathcal{f}$ Epidemiol 1995;142:331-41.

5 Quinn TC, Glasser D, Cannon RO. Human immunodeficiency virus infection among patients attending clinics deficiency virus infection among patients attending clinics
for sexually transmitted diseases. $N$ Engl f Med 1988;318: 197-203.

6 Muga R, Roca J, Tor J, et al. Syphilis in injecting drug users: clues for the high-risk sexual behaviour in females IDUs. Int f STD AIDS 1997;8:225-8.

7 Metzger DS, Woody GE, McLellan AT, et al. Human immunodeficiency virus seroconversion among intravenous drug users in- and out-of-treatment: a 18-month prospective follow-up. F Acquir Immune Defic Syndr 1993;6: 1049-56.

8 Holmberg SD. The estimated prevalence and incidence of HIV in 96 large US metropolitan areas. Am f Public Health 1996;86 642-54.

9 Monterroso E, Prevots R. East vs West coast differences in HIV seroincidence and behavioral risk factors for incident HIV infection among street- and drug-treatment recruited injection drug users. 124th Annual Meeting of the Ameriinjection drug users. 124th Annual Meeting of the Ameri-
can Public Health Association, November, New York, 1996.

10 St Louis ME. Strategies for syphilis prevention in the 1990s. Sex Transm Dis 1996;23:58-67.

11 Schoefer H, Imhof M, Thoma-Greber E, et al. Active syphilis in HIV infection: a multicentre retrospective survey. Genitourin Med 1996;72:176-81.

12 Ansell DA, Hu TC, Straus M, et al. HIV and syphilis seroprevalence among clients with sexually transmitted diseases attending a walk-in clinic at Cook County Hospital. Sex Transm Dis 1994;21:93-6.

13 Siegal HA Carlson RG, Flack R, et al. HIV infection and risk behaviors among intravenous drug users in low prevalence Friedman SR, Des Jarlais DC. HIV among drug injectors: the epidemic and the response. AIDS Care 1991;3:239-50. 15 Kerndt P, Weber M, Ford W, et al. HIV incidence among Kerndt $P$, Weber $M$, Ford $W$, et al. HIV incidence among
injection drug users enrolled in a Los Angeles methadone injection drug users enrolled in a
program. $¥ A M A 1995 ; 273: 1831$.

program. $\mathcal{F A M A}$ 1995;273:1831.
16 Kilmarx PH, Louis ME. Editorial: The evolving epidemiolKilmarx PH, Louis ME. Editorial: The evolving epidem
ogy of syphilis. Am $\mathcal{F}$ Public Health 1995;85:1053-4.

17 Battjes RJ, Pickes RW, Amsel Z. Introduction of HIV infection among intravenous drug abusers in low prevalence areas. F Acquir Inmune Defic Syndr 1989:2:533-9.

18 Division of STD Prevention (US Department of Health and Human Services, Public Health Service). Sexually Transmitted Disease Surveillance, 1995. Atlanta, Sept 1996.

19 California Department of Health Services. County Health Status Profiles, 1996.

20 Los Angeles County Department of Health Services. Communicable Disease Morbidity Report, 1993. Los Angeles, 1995.

21 Centers for Disease Control. Case definitions for public health surveillance. MMWR Morb Mort Wkly Rep 1990; 39(RR-13): 1-43.

22 Gourevitch MN, Hartel D, Schoenbaum EE, et al. A prospective study of syphilis and HIV infection among injection drug users receiving methadone in the Bronx, NY. injection drug users receiving methado

23 Siegel D, Larsen SA, Golden E, et al. Prevalence, incidence, and correlates of syphilis seroreactivity in multiethnic San Francisco neighborhoods. Ann Epidemiol 1994;4:460-5.

24 Centers for Disease Control. Selective screening to augment syphilis case-finding-Dallas, 1991. MMWR Morb Mort Wkly Rep 1993;42:424-7.

25 Flores JL. Syphilis. A tale of twisted treponemes. West f Med 1995;163:552-9.

26 Centers for Disease Control. Primary and secondary syphilis-United States, 1981-1990. MMWR Morb Mort Wkly Rep 1991;40:314-23.

27 Williams ML, Elwood WN, Weatherby NL, et al. An assessment of the risk of syphilis and HIV infection among a sample of not-in-treatment drug users in Houston, Texas. AIDS Care 1996;8:671-82.

28 Chaisson RE, Moss AR, Onishi R, et al. Human immunodeficiency virus infection in heterosexual intravenous drug users in San Francisco. Am $\mathcal{F}$ Public Health 1987;77:16972 .

29 Watters JK, Estilo MJ, Kral AH, et al. HIV infection among female injection-drug users recruited in community settings. Sex Transm Dis 1994;21:321-8. 
30 Lewis DK, Watters JK. Sexual risk behaviors among heterosexual intravenous drug users: ethnic and gender variasexual intravenous drug use
tions. AIDS 1991;5:77-83.

31 Booth RE, Watters JK, Chitwood DD. HIV risk-related sex behaviors among injection drug users, crack smokers, and injection drug users who smoke crack. Am f Public Health 1993;83:1144-8.

32 Chaisson RE, Baccheti, Osmond D, et al. Cocaine use and HIV infection in intravenous drug users in San Francisco. ҰAMA 1989;261:561-5.

33 Otten MW, Zaidi AA, Peterman TA, et al. High rates of HIV seroconversion among patients attending urban sexually transmitted disease clinics. AIDS 1994;8:549-53.

34 Thomas DL, Cannon RO, Shapiro CN, et al. Hepatitis C, hepatitis $\mathrm{B}$, and human immunodeficiency virus infections among non-intravenous drug-using patients attending clinics for sexually transmitted diseases. F Infect Dis 1994;169: 1cs for sext

35 Herrera GA, Lackritz EM, Janssen RS, et al. Serologic test for syphilis as a surrogate marker for human immunodeficiency virus infection among United States blood deficiency virus infection among
donors. Transfusion 1997;37:836-40.

36 Wilson TE, Howard M, DeHovitz J, et al. The relationship of cocaine use and human immunodeficiency virus serostatus to incident sexually transmitted diseases among women. Sex Transm Dis 1998;25:70-5.
37 Friedman SR, Jose B, Deren S, et al. Risk factors for human immunodeficiency virus seroconversion among out of treatment drug injectors in high and low seroprevalence cities. Am f Epidemiol 1995;142:864

38 Nelson KE, Vlahov D, Cohn S, et al. Sexually transmitted diseases in a population of intravenous drug users: association with seropositivity to the human immunodeficiency virus (HIV). F Infect Dis 1991;164:457-63.

39 Scribner R, Cohen D, Dwyer J. Smokeable cocaine use and risk of sexually transmitted disease among male inmates. Sixth International Conference on AIDS, 1990. San Francisco, CA, 1990.

40 Hser Y. Population estimates of intravenous drug users and HIV infection in Los Angeles County. Int f Addict 1993;28: 695-709.

41 Nandwani R, Evans DTP. Are you sure it's syphilis? A review of false positive serology. Int $\mathcal{f}$ STD AIDS 1995;6:241-8

42 Thomas DL, Quinn TC. Serologic testing for sexually transmitted diseases. Sexually transmitted diseases in the AIDS era. Infect Dis Clin N Am 1993;7:793-824.

43 Barthwell AG, Gibert CL. Screening for infectious diseases among substance abusers. Rockville, MD: US Department of Health and Human Services, 1993. (Treatment Improvement Protocol 6.) 